\title{
The sum of induced and real motion is not a straight path
}

\author{
ROBERT B. POST and MARC CHADERJIAN \\ University of California, Davis, California
}

\begin{abstract}
The apparent motion path of a stimulus undergoing simultaneous real motion and orthogonal induced motion (IM) was measured by having subjects draw the perceived path. The average path was not a straight line, but was curved away from the direction of inducer motion. This result is consistent with a previous finding that IM magnitude increases with stimulus duration, and with a theory that attributes IM to the suppression of reflexive eye movements. Implications are discussed for research in which the apparent slope of the motion path of a stimulus undergoing simultaneous IM and real motion is used as a measure of IM.
\end{abstract}

Induced motion (IM) is the illusory motion of a visual stimulus that occurs opposite the direction of motion of other visual detail. Since the early report of this phenomenon by Duncker (1929), a variety of stimuli and response measures have been employed to investigate the amount of IM that occurs under various conditions. One convenient technique for the quantification of IM involves the addition of real motion in a direction perpendicular to the direction of IM. The IM and real motion vectors combine to produce apparent motion on a slant, the slope of which may be used to derive the magnitude of the IM component (see, e.g., Gogel \& Tietz, 1976, or Wallach, Bacon, \& Schulman, 1978). We will refer to this technique for measurement of IM as the slant matching method.

Recently, Post and Heckman (1986) used the slant matching method to measure the amount of IM during prolonged viewing of an IM display containing unidirectional inducing stimulus motion, and discovered that IM increased as a function of inducer duration. This finding leads to a prediction concerning the apparent path of a stimulus undergoing simultaneous real motion and IM. Specifically, because the magnitude of the IM vector is gradually increasing, a stimulus containing both IM and orthogonal real motion should appear to move first primarily in the direction of its real motion component, and then increasingly curve in a direction opposite the direction of inducer motion as the magnitude of the IM vector increases. In the present paper we investigated this possibility by having subjects both describe and draw the apparent motion path of an IM stimulus that contained a real motion vector orthogonal to the direction of inducing motion.

This research was supported by University of California Grant D-1832 to R.B.P. We are grateful to Chris Johnson for the loan of a function generator and to Tom Heckmann for a critical reading of the manuscript. We are also grateful for the helpful comments of two anonymous reviewers of the manuscript. Address correspondence to Robert B. Post, Department of Psychology, University of California, Davis, CA 95616 .
An additional concern in the present experiment was to determine the generality of the previously cited effect of inducer duration on IM. Specifically, the study in which duration was found to influence IM magnitude (Post \& Heckmann, 1986) employed a large, unidirectionally moving inducing stimulus with a velocity of either 5 or $60 \mathrm{deg} / \mathrm{sec}$. Although such an inducer was useful for investigating the changes in IM during prolonged stimulation, the inducers commonly used in IM research are typically smaller and oscillatory. Therefore, a small, slow, oscillatory inducer was used in the present experiment with the aim of determining whether the effect of duration on IM (as indicated in the present study by the predicted curvature effect) extends to this class of inducing stimuli as well.

\section{METHOD}

IM stimuli were formed by attaching a function generator to adjacent oscilloscopes to create horizontal oscillation $(.125 \mathrm{~Hz}$, triangular waveform) of a dot on the right scope (the inducing stimulus) and vertical oscillation of a dot on the left scope. Rightward and leftward movements of the right dot were paired with upward and downward movements, respectively, of the left dot. The midpoint of vertical motion of the left dot corresponded to the level of the right dot. There was a separation of $4.6^{\circ}$ between the vertical motion path and the leftmost position of the right dot (see Figure 1 for the physical paths of the stimuli). Motion of the dots was of constant velocity $(1.15 \mathrm{deg} / \mathrm{sec})$ through an angle of $4.6^{\circ}$ per half cycle. Intensity of the stimuli was adjusted so no other detail was visible.

Ten volunteers, aged 9 to 40 years, with corrected Snellen acuity of at least 20/20 OU served as subjects. All observations were binocular; the subject was seated and head position and viewing distance of $75 \mathrm{~cm}$ were maintained by means of a chinrest. The subjects were instructed to fixate (track) the left, vertically moving dot and to attend to the apparent path of its motion. When they were satisfied that they could reproduce the apparent motion path of the tracked dot, the subjects drew the perceived path on graph paper on which the horizontal and vertical axes of motion were clearly labeled. There were no marks on the paper to constrain the size of the drawing. Subjects were also asked to describe whether the path of the tracked stimulus appeared straight or curved, and, if curved, the direction of curvature. Three replications of both the 

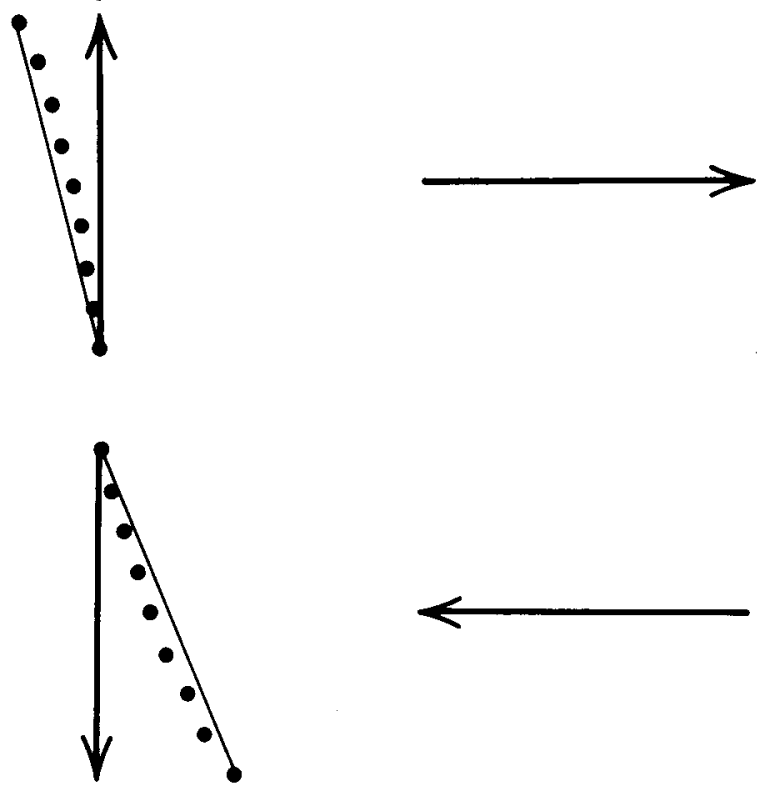

Figure 1. Mean path of a stimulus undergoing simultaneous vertical real motion and horizontal induced motion. Top panel: Apparent path of tracked stimulus while moving upward. Bottom panel: Apparent path of tracked stimulus while moving downward. Dots correspond to the perceived motion path of the tracked stimulus. Real motion of the inducing and tracked stimuli is represented by the right and left solid arrows, respectively. The thin diagonal solid lines connect the beginning and end points of the motion path drawn by subjects, and provide a straight line for comparison to the obtained data.

drawing and verbal responses were obtained for each subject for both directions of motion. The tracked dot oscillated continuously vertically. Half of the subjects made judgments first for upward motions and the other half made judgments first for downward motions.

A second group of 10 individuals, aged 18 to 37 years, with corrected Snellen acuity of at least $20 / 20$ OU were subjects in a control condition in which similar measures were obtained in the absence of the inducing stimulus. Except for the fact that the inducing stimulus was not present, the method for this group was identical to that described for the experimental group. Results obtained from this condition provided a baseline for ascertaining that reports of slanted and curved perceived motion paths in the experimental condition were dependent on the presence of an inducing stimulus.

\section{RESULTS}

Measures obtained without the inducing stimulus indicated that the perceived motion path of the solitary tracked dot was typically not slanted. On the 30 trials for upward motion, 24 paths were drawn and described as moving vertically (the endpoint of motion was directly above the starting point) and 6 were drawn and described as moving up and to the right. On the 30 trials for downward motion, 20 paths were drawn and described as moving vertically, 7 as moving down and to the right, and 3 as moving down and to the left. The path was also typically not reported as curved. On the 30 trials for upward mo- tion, 24 paths were drawn and described as straight, 1 as appearing to curve toward the left while moving up, and 5 as being increasingly curved to the right. On the 30 trials for downward motion, 21 paths were drawn and described as straight, 6 as appearing to curve toward the left, and 3 as appearing to curve to the right. The apparent direction and curvature of the path of the tracked stimulus in the absence of an inducer therefore tended to be veridical, with some nonsystematic variability. This pattern would be expected if some degree of autokinesis is perceived in addition to the veridical motion path.

Measures obtained in the experimental condition, in which the inducing stimulus was present, contrast sharply with the above pattern. Both the verbal descriptions and the drawings of the perceived path of the tracked stimulus indicated that the experimental conditions generated IM. Each of the 10 subjects drew at least one path for each direction of inducer motion that indicated that the tracked stimulus appeared to move opposite the inducer. On the 30 trials with rightward inducer motion, 25 drawings indicated that the upward-moving tracked stimulus appeared to move up and to the left (i.e., displayed IM). On the 30 trials with leftward movement of the inducing stimulus, 29 drawings indicated that the downward-moving tracked stimulus appeared to move down and to the right. None of the 60 responses indicated perceived motion of the tracked stimulus in the same direction as the inducer, that is, opposite to the expected direction of IM. The verbal descriptions provided by the subjects corresponded precisely to the data obtained with the drawing measure.

The frequencies of vertical, leftward, and rightward perceived motion obtained with the inducer stimulus were compared statistically with those reported without an inducer. Separate chi-square analyses performed for the upward and downward trials revealed a highly significant influence of the inducer on the direction of the perceived motion path $\left[\chi^{2}(2)=43.4, p<.001\right.$, and $\chi^{2}(2)=33.6$, $p<.001$, for upward and downward trials, respectively].

The prediction that the path of a stimulus undergoing simultaneous IM and orthogonal real motion should appear curved away from the inducer motion was also supported by both descriptions and drawings of the perceived paths. On the 25 trials in which IM was present for an upward-moving tracked stimulus, 18 paths were both described and drawn as curving to the left (away from the direction of inducer motion). This direction of curvature is consistent with the finding that the magnitude of IM increases while the inducer is moving in one direction. None of the responses indicated curvature that would be consistent with decreasing IM. Similarly, on the 29 trials in which IM was present during downward motion of the tracked stimulus, 17 paths were both described and drawn as curving to the right, indicating an increasing IM vector during downward motion. Again, none of the responses indicated curvature in a direction consistent with a decreasing IM vector. These results were significantly different from those obtained in the control condition 
$\left[\chi^{2}(2)=20, p<.001\right.$, and $\chi^{2}(2)=19.5, p<.001$, for upward and downward trials, respectively].

To evaluate the degree of curvature present in the subjects' drawings of the motion path of the tracked stimulus, the vertical extent of each response was first normalized to a unit value. The horizontal deviation of the drawn path from vertical was then computed for each response as a proportion of the total vertical extent for each of nine locations equally spaced along the vertical axis of stimulus motion. The mean horizontal deviation at each location was computed separately for the $\mathbf{3 0}$ upward and downward trials; these values are presented in Figure 1.

The curvature of the perceived path of a stimulus undergoing simultaneous IM and real motion is apparent in the figure. In the case of upward stimulus movement, the tracked dot appeared at first to move primarily in the upward direction (in the direction of its real-motion component) and then to curve in the direction opposite to the inducing dot (in the direction of IM). In the bottom portion of the figure, a similar pattern is observed for the downward-moving dot. Specifically, it appeared to move first primarily downward and then to curve to the right (in the direction of IM). It is also apparent in the figure that the magnitude of IM obtained during downward motion of the tracked stimulus was larger than that obtained during upward motion.

\section{DISCUSSION}

The primary finding of this study was that a stimulus undergoing simultaneous IM and orthogonal real motion appeared to travel on a curved rather than a straight path. The apparent curvature was obtained for both directions of motion of a vertically oscillating tracked stimulus, and the opposite curvature was never reported. The curvature corresponded to an increase in the perceived magnitude of the IM vector while the stimulus was moving in one direction.

The apparent curvature of a stimulus undergoing simultaneous IM and orthogonal real motion is consistent with reports that the magnitude of IM increases during prolonged viewing of an $\mathrm{IM}$ display containing a unidirectional inducer (Heckmann \& Post, 1986; Post \& Heckmann, 1986). In those studies, the magnitude of IM was sampled every $5 \mathrm{sec}$ from continuous records of judgments during $2 \mathrm{~min}$ of unidirectional inducer motion. IM gradually increased in magnitude during the initial portion of the 2-min exposure and then reached an asymptotic value. The curvature of the perceived motion path in the present study indicates that the increase in IM with increasing inducer duration may also be observed during the $4 \mathrm{sec}$ in which the oscillating stimulus was travelling in one direction. Additionally, the inducer used in the present study was small and of low velocity, thereby extending the previously reported findings of duration effects to most of the range of inducing stimuli employed in IM research.
The increase in IM obtained in the present study is consistent with the hypothesis that IM results in part from the interaction of two smooth eye-movement systems in the maintenance of fixation (Post \& Heckmann, 1986; Post \& Leibowitz, 1985; Post, Shupert, \& Leibowitz, 1984). Briefly, this theory posits that motion of the inducing stimulus in IM displays activates a reflexive eyemovement system (optokinetic nystagmus, or OKN), which normally serves to stabilize gaze on the environment during self-motion. In order to maintain fixation on the stimulus that has had motion induced in it, it is therefore necessary to oppose the activity in the OKN system with activity in the smooth-pursuit eye-movement system in the opposite direction. The proposal that smooth pursuit suppresses OKN is consistent with a wide variety of neurological findings indicating that disruption of the smooth-pursuit system also impairs the ability to suppress nystagmus (see, e.g., Dichgans, von Reutern, \& Rommelt, 1978; Takemori \& Cohen, 1974). Activity in the smooth-pursuit system gives rise to the perception that the fixated stimulus is moving in the direction of the pursuit activity, that is, IM. This theory predicts the present finding that IM magnitude increases with the duration of the inducing stimulus. This follows from the fact that the OKN system is activated gradually by moving visual contours (Cohen, Henn, Raphan, \& Dennett, 1981; Cohen, Matsuo, \& Raphan, 1977), with its activity building up slowly to an asymptotic level during prolonged stimulation. Because OKN activation builds up slowly, the smooth-pursuit activity necessary to oppose the reflex should also increase gradually during viewing of an IM display and, accordingly, so should IM.

A different explanation for IM emphasizes the relative motion of elements of the visual display (Duncker, 1929; Mack, Heuer, Fendrich, Vilardi, \& Chambers, 1985). It is not apparent that the present finding of perceived curvature of the motion path would be expected from this theoretical approach. The relative motion between the elements of the display remains constant during each period in which the stimuli are moving in one direction, yet IM increases during this period to produce apparent curvature.

A third explanation for IM posits that motion of the inducing stimulus causes displacement of the subject's apparent straight-ahead (ASA) in the direction of inducer motion, and thereby produces apparent opposite motion of other details (Bridgeman \& Klassen, 1983; Brosgole, 1968). The present findings provide neither support for nor opposition to this particular theory, inasmuch as ASA was not measured.

We noted previously that the magnitude of IM during downward motion of the tracked stimulus (and leftward motion of the inducer) was greater than that during upward motion. A possible explanation of this finding is that during downward motion of the tracked stimulus, the inducer was approaching the tracked stimulus, whereas in the case of upward motion of the tracked stimulus, the inducer was moving away. Gogel (1974) reported that spa- 
tial separation of similar inducing and induced stimuli influences the magnitude of IM. Specifically, more IM is obtained with spatially adjacent stimuli than with remote stimuli. In the present study, therefore, the effects of stimulus duration on IM and the effect of spatial separation on IM were additive for the case of the downwardmoving tracked stimulus. That is, as IM was increasing during the latter portion of the downward motion, the inducer was also becoming more effective as a result of its decreased spatial separation. It should be noted that the increase of IM with decreased spatial separation is also predicted by the oculomotor theory of IM. Specifically, the effectiveness of a stimulus for activating the OKN system varies inversely with retinal eccentricity (Cheng \& Outerbridge, 1975). Therefore, as the tracked and inducing stimuli approach each other, more activation of the OKN system occurs, and more pursuit effort must be exerted to suppress nystagmus. Therefore, IM magnitude increases (Post et al., 1984).

The influence of duration on IM magnitude obtained in the present study has potential implications for the study of the influence of stimulus velocity on IM magnitude. It has previously been reported (Becklen \& Wallach, 1985; Wallach \& Becklen, 1983) that IM magnitude decreases with increased velocity of the inducing stimulus. In contrast to these reports, Post and Heckmann (1986) reported greater IM with stimulation of $60 \mathrm{deg} / \mathrm{sec}$ than with $5 \mathrm{deg} / \mathrm{sec}$, and Post (1986) reported linear increases in IM velocity up to $180 \mathrm{deg} / \mathrm{sec}$. A resolution of these findings is suggested by the present findings and the previous report (Post \& Heckmann, 1986) of the influence of inducer duration on IM. Specifically, the inducer employed by Wallach and Becklen (1983) and Becklen and Wallach (1985) oscillated, so stimulus velocity varied inversely with stimulus duration. It is to be expected that the brief durations of high-velocity inducingstimulus motions used in these studies would have little influence on the OKN system; accordingly, little IM should result. In the studies of Post and Heckmann (1986) and Post (1986), however, the inducer moved at a relatively high velocity in only one direction, thereby allowing sufficient duration of motion for activation of the OKN system. Correspondingly, IM occurred as OKN activity was opposed.

The present study also has practical implications for research in which the slant matching method is employed to measure IM. Specifically, care should be taken to ensure that there is consistency with regard to the portion of the motion path subjects are attempting to match. At- tempts to match the initial slant of the motion path will indicate less IM than will judgments based on the final portion of stimulus motion in any one direction.

\section{REFERENCES}

BECKLEN, R., \& WALLACH, H. (1985). How does speed change affect induced motion? Perception \& Psychophysics, 37, 231-236.

Bridgeman, B., \& Klassen, H. (1983). On the origin of stroboscopic induced motion. Perception \& Psychophysics, 34, 149-154.

Brosgole, L. (1968). An analysis of induced motion. Acta Psycholog ica, 28, 1-44.

Cheng, M., \& OUterbridge, J. S. (1975). Optokinetic nystagmus during selective retinal stimulation. Experimental Brain Research, 23, 129-139.

Cohen, B., Henn, V., Raphan, T., \& Dennett, D. (1981). Velocity storage, nystagmus, and visual-vestibular interactions in humans. $A n-$ nals of the New York Academy of Sciences, 374, 421-433.

Cohen, B., Matsuo, V., \& RaPhan, T. (1977). Quantitative analysis of the velocity characteristics of optokinetic nystagmus and optokinetic after-nystagmus. Journal of Physiology (London), 270, 321-344.

Dichgans, J., von Reurern, G. M., \& Rommelt, U. (1978). Impaired suppression of vestibular nystagmus by fixation in cerebellar and noncerebellar patients. Archiv für Psychiatrie und Nervenkrankheiten, 226, 183-199.

Duncker, K. (1929). Uber induzierte Bewegung. Psychlogische Forschung, 12, 180-259.

GOGEL, W. C. (1974). Relative motion and the adjacency principle. Quarterly Journal of Experimental Psychology, 26, 425-437.

GoGel, W. C., \& TieTZ, J. D. (1976). Adjacency and attention as determinants of perceived motion. Vision Research, 16, 839-845.

HeckmanN, T., \& Post, R. B. (1986). Induced motion and apparent straight ahead during prolonged stimulation. Investigative Ophthalmology \& Visual Science, 27(Suppl. 3), 346.

Mack, A., Heur, F., Fendrich, R., Vilardi, K., \& Chambers, D. (1985). Induced motion and oculomotor capture. Journal of Experimental Psychology: Human Perception \& Performance, 11, 329-345.

Post, R. B. (1986). Induced motion considered as a visually induced oculogyral illusion. Perception, 15, 131-138.

Post, R. B., \& HeckmanN, T. (1986). Induced motion and apparent straight ahead during prolonged stimulation. Perception \& Psychophysics, 40, 263-270.

Post, R. B., \& Leibowitz, H. W. (1985). A revised analysis of the role of efference in motion perception. Perception, 14, 631-643.

Post, R. B., Shupert, C. L., \& Leibowitz, H. W. (1984). Implications of OKN suppression by smooth pursuit for induced motion. Perception \& Psychophysics, 36, 493-498.

TAKEMORI, S., \& COHEN, B. (1974). Loss of visual suppression of vestibular nystagmus after flocculus lesions. Brain, 72, 213-224.

Wallach, H., Bacon, J., \& Schulman, P. (1978). Adaptation in motion perception: Alteration of induced motion. Perception \& Psychophysics, 24, 509-514.

WALLACH, H., \& BECKLEN, R. (1983). An effect of speed on induced motion. Perception \& Psychophysics, 34, 237-242.

(Manuscript received March 27, 1987; revision accepted for publication August 5, 1987.) 